\title{
Electronic structure and magnetic interactions of the spin-chain compounds $\mathrm{Ca}_{2} \mathrm{CuO}_{3}$ and $\mathrm{Sr}_{2} \mathrm{CuO}_{3}$
}

\author{
Coen de Graaf and Francesc Illas \\ Departament de Química Física i Centre de Recerca en Química Teòrica, Universitat de Barcelona, C/Martí i Franquès 1, \\ 08028 Barcelona, Spain
}

(Received 23 June 2000; published 11 December 2000)

\begin{abstract}
The results are presented of a combined periodic and cluster model approach to the electronic structure and magnetic interactions in the spin-chain compounds $\mathrm{Ca}_{2} \mathrm{CuO}_{3}$ and $\mathrm{Sr}_{2} \mathrm{CuO}_{3}$. An extended $t-J$ model is presented that includes in-chain and interchain hopping and magnetic interaction processes with parameters extracted from $a b$ initio calculations. For both compounds, the in-chain magnetic interaction is found to be around -240 $\mathrm{meV}$, larger than in any of the other cuprates reported in the literature. The interchain magnetic coupling is found to be weakly antiferromagnetic, $-1 \mathrm{meV}$. The effective in-chain hopping parameters are estimated to be $\sim 650 \mathrm{meV}$ for both compounds, whereas the value of the interchain hopping parameter is $30 \mathrm{meV}$ for $\mathrm{Sr}_{2} \mathrm{CuO}_{3}$ and $40 \mathrm{meV}$ for $\mathrm{Ca}_{2} \mathrm{CuO}_{3}$, in line with the larger interchain distance in the former compound. These effective parameters are shown to be consistent with expressions recently suggested for the Néel temperature and the magnetic moments, and with relations that emerge from the $t$ - $J$ model Hamiltonian. Next, we investigate the physical nature of the band gap. Periodic calculations indicate that an interpretation in terms of a chargetransfer insulator is the most appropriate one, in contrast to the suggestion of a covalent correlated insulator recently reported in the literature.
\end{abstract}

DOI: 10.1103/PhysRevB.63.014404

PACS number(s): 75.30.Et, 74.25.Ha, 74.25.Jb, 71.15.-m

\section{INTRODUCTION}

The one-dimensional antiferromagnetic spin-1/2 chain has received considerable theoretical attention being a relatively simple yet nontrivial quantum mechanical model. $\mathrm{Ca}_{2} \mathrm{CuO}_{3}$ and $\mathrm{Sr}_{2} \mathrm{CuO}_{3}$ are generally considered to be the best experimental realizations of one-dimensional (1D) antiferromagnetic (AF) spin-1/2 chains, and hence, very interesting candidates to test the wide range of theoretical predictions reported in the literature. The exact solution of the Heisenberg model Hamiltonian for the AF spin-1/2 chain $^{1}$ is a singlet (i.e., $S=0$ ) and exhibits a vanishing long-range order. The low-lying excited states have been interpreted as spinwave like states with $S=1,{ }^{2}$ being a superposition of two spin-1/2 objects. ${ }^{3}$ The extremely low Néel temperature $T_{N}$ combined with the very small magnetic moment measured for $\mathrm{Ca}_{2} \mathrm{CuO}_{3}$ and $\mathrm{Sr}_{2} \mathrm{CuO}_{3},{ }^{4-6}$ are indeed in line with the character of the Bethe solution and the prediction for the low-lying spin excitations has been confirmed in inelastic neutron scattering experiments for $\mathrm{KCuF}_{3}{ }^{7}$

Furthermore, there exists a wealth of numerical studies that explore the dynamics of the 1D Heisenberg system. A very relevant contribution to the understanding of the spin chains was made by Bonner and Fisher, ${ }^{8}$ who estimated numerically the magnetic susceptibility as a function of the temperature. The Bonner-Fisher curve has rather recently been revised by Eggert, Affleck, and Takahashi ${ }^{9}$ to improve the correspondence with the experimentally observed susceptibility at low temperatures. Moreover, the nuclear magnetic resonance (NMR) relaxation rates observed in $\mathrm{Sr}_{2} \mathrm{CuO}_{3}$ have been successfully interpreted with the use of Quantum Monte Carlo techniques ${ }^{10,11}$ and a recent combined analytical and numerical study of Lorenzana and Eder ${ }^{12}$ describes in detail the phonon-assisted magnetic excitations in the 1D spin-1/2 chain.
All these studies have in common that the underlying Heisenberg Hamiltonian is strictly one dimensional and only consider the in-chain magnetic interactions. Despite the success of this model Hamiltonian, it cannot account for the finite temperature at which 3D ordering sets in, and hence, additional magnetic interactions need to be introduced in the model. The first attempt to relate the Néel temperature with the interchain interactions in $\mathrm{Sr}_{2} \mathrm{CuO}_{3}$ was given by Ami et $a l^{5}$ Assuming dipolar interactions only, an extremely small Néel temperature was obtained, and therefore, the authors conclude that other than dipolar interactions, e.g., direct interchain hopping, ${ }^{13}$ play a significant role. Recently various proposals have been published that go beyond the simple expression used by Ami et al. In the framework of a mean-field treatment of the interchain interactions, Schulz has proposed a theory to relate the strength of the interchain interaction with the Néel temperature. ${ }^{14}$ The relation applies reasonably well both for $\mathrm{KCuF}_{3}$ and $\mathrm{Sr}_{2} \mathrm{CuO}_{3}$, although the latter compound has interchain interactions along the two directions perpendicular to the spin chain that differ by orders of magnitude, while only one (effective) interchain interaction appears in the theoretical model. An extension of the mean-field theory has been developed recently by Sandvik. ${ }^{15}$ The interactions between the central chain and its neighbors are treated as exactly as possible or simulated with Quantum Monte Carlo techniques, while the rest of the crystal is represented by a staggered magnetic field, i.e., a meanfield treatment. In contrast to the expressions derived by Schulz, this approach naturally accounts for the existence of interchain interactions of different strengths. An alternative way to improve the mean-field result is to add corrections obtained within a $1 / z_{\perp}$ expansion, ${ }^{16}$ with $z_{\perp}$ the number of nearest neighbor chains. These corrections significantly improve the overestimation of $T_{N}$ generally obtained within the mean-field treatment. ${ }^{14,17}$ 
The success of all these models depends critically on the reliability of the input parameters. For the strictly 1D models there is only one adjustable parameter, the in-chain magnetic interaction parameter $J$, while the models aimed at getting information about the order/disorder transition need additional interchain interaction parameters. However, there seems to be no consensus about the magnitude of these parameters. For example, the determination of the in-chain magnetic coupling parameter by fitting the magnetic susceptibility data of $\mathrm{Sr}_{2} \mathrm{CuO}_{3}$ have resulted in rather different values, ranging from $-146 \mathrm{meV},{ }^{18}$ to $-190 \mathrm{meV},{ }^{19}$ and to $-225 \mathrm{meV}{ }^{5}$ Considering other estimates of $J$, the dispersion becomes even larger: -130 to $-160 \mathrm{meV}$ from angleresolved photoemission spectroscopy (ARPES) ${ }^{20}$ and -246 to $-260 \mathrm{meV}$ from the description of the phonon-assisted magnetic excitations appearing in the midinfrared spectrum of $\mathrm{Sr}_{2} \mathrm{CuO}_{3} \cdot{ }^{12,21}$ Finally, ${ }^{63} \mathrm{Cu}$ NMR data indicate that the magnitude of $J$ is around $240 \mathrm{meV} .^{11,22}$ No direct experimental data for the interchain interactions exist. The only estimates reported in the literature are exactly those derived from the equations that relate the Néel temperature with such interactions. ${ }^{13,14,16}$ In summary, the dispersion of the values for the in-chain magnetic coupling parameter and the lack of independent estimates for the interchain interaction strengths, makes necessary an accurate alternative determinations of these parameters.

Beside the information about the magnetic behavior of the (quasi-)1D antiferromagnetic spin-1/2 chains, the electronic structure of these compounds is also of interest. $\mathrm{Sr}_{2} \mathrm{CuO}_{3+\delta}$ is one of the few examples of a cuprate compound that exhibits superconductivity $\left(T_{c} \approx 70 \mathrm{~K} \text {, under high pressure }\right)^{23}$ but does not possess the typical $\mathrm{CuO}_{2}$ planes. The study of the electronic structure, either by first principles calculations or by spectroscopic techniques, not only provides a qualitative description of the ground state but also important information about the character of the gap, charge-transfer (CT) energy, effective hopping integrals $(t)$, and other relevant parameters. Two studies concerning the band structure of $\mathrm{Sr}_{2} \mathrm{CuO}_{3} / \mathrm{Ca}_{2} \mathrm{CuO}_{3}$ have been published. In the first place, Rosner et al. have reported local density approximation (LDA) calculations for the two cuprates. ${ }^{13}$ From a careful analysis of available experimental data and their LDA results, various hopping and magnetic coupling parameters are derived. However, it must be noted that LDA predicts the compounds to be metallic, while the experimental band gaps are of the order of $2 \mathrm{eV} .{ }^{24,25}$ This well-known artifact of $\mathrm{LDA}^{26-28}$ results in exceedingly large magnetic coupling parameters, ${ }^{29,30}$ and the consequences on the hopping parameters will be discussed at length in this paper. Second, a combined approach of various electron spectroscopy measurements and semiempirical many body calculations is reported by Maiti and co-workers. ${ }^{25}$ Apart from a detailed analysis of the size of the band gap $\left(1.5 \mathrm{eV}\right.$ for $\mathrm{Sr}_{2} \mathrm{CuO}_{3}$ and $1.7 \mathrm{eV}$ for $\mathrm{Ca}_{2} \mathrm{CuO}_{3}$ ), the most prominent conclusion of this work is that $\mathrm{Sr}_{2} \mathrm{CuO}_{3}$ is predicted to be a so-called correlated covalent insulator, i.e., the upper Hubbard band (empty $\mathrm{Cu} d$ levels) overlaps with the $\mathrm{O} 2 p$ band. This is in contrast with the generally accepted interpretation of the (undoped) latetransition metal oxides as CT insulators. ${ }^{31,32}$
Therefore, there are two main points that we want to address in this paper: First, the strength of the different magnetic coupling parameters in these spin chain compounds and second, a study of the band structure in order to clarify the physical character of the band gap. For this purpose we have performed an $a b$ initio study combining the local cluster approach with periodic band structure calculations. This combination allows us to obtain complementary information about the electronic structure of the compounds under study. After the pioneering work of Wachters and Nieuwpoort in the early 1970 's, ${ }^{33}$ the application of modern $a b$ initio quantum chemical techniques within the cluster approach to calculate magnetic interaction strengths in ionic insulators expanded enormously in the last $10 \mathrm{yr}$. It now provides a welldocumented field and has been proven to accurately reproduce experimental data, ${ }^{34,35}$ and hence allows us to make predictions of $J$ for materials for which experimental data are absent, scarce, or contradictory. ${ }^{36-39}$ Nearly all first principles periodic electronic structure calculations for transition metal oxides are based on the density functional theory (DFT), in which the LDA is applied to express the unknown exchange-correlation functional. Self interaction corrected $\mathrm{LDA}^{40}$ and LDA $+U^{41,42}$ seem to correct for the shortcomings of LDA, but the fact that $U$ can be adjusted to best fit experimental data ${ }^{43}$ makes the $a b$ initio character of the method somewhat questionable. ${ }^{44}$ Here, we opt for ab initio periodic unrestricted Hartree-Fock (UHF) calculations ${ }^{45,46}$ and compare the results to those obtained from density functional calculations applying the $\mathrm{B} 3 \mathrm{LYP}^{47}$ and the F-B:LYP functionals for the exchange-correlation part of the density functional. B3LYP is widely used in quantum chemical calculations and is extremely successful in reproducing a wide variety of molecular properties. The F-B:LYP functional has been taken for its ability to predict rather accurately magnetic coupling parameters in ionic insulators as shown by Martin and Illas. ${ }^{29,30}$ Section II B gives more detailed information about the functionals applied. The purpose of the periodic calculations is twofold. In the first place, we calculate the band structure of both compounds, and second, the comparison of cluster and periodic calculations at the same level of approximation of the $\mathrm{N}$-electron wave function allows us to verify the reliability of the material model applied in the cluster calculations. ${ }^{48,49}$

The remaining part of the paper is organized as follows. Section II shortly discusses the crystal structure of the two compounds and gives a detailed description of the computational strategy used in the cluster and periodic calculations. In Sec. III, we first discuss the magnetic coupling parameters. Attention will be focused on the quality of the $\mathrm{N}$-electron wave function and on the appropriateness of the cluster model. Results of cluster and periodic calculations are compared. Thereafter, a discussion of the electronic structure will be given. We carefully inspect the character of the band gap and the size of different effective hopping parameters. After analyzing the behavior of some spin-polarized computational schemes, we examine the consistency of the parameters derived. The paper concludes with a short summary of the most relevant points. 


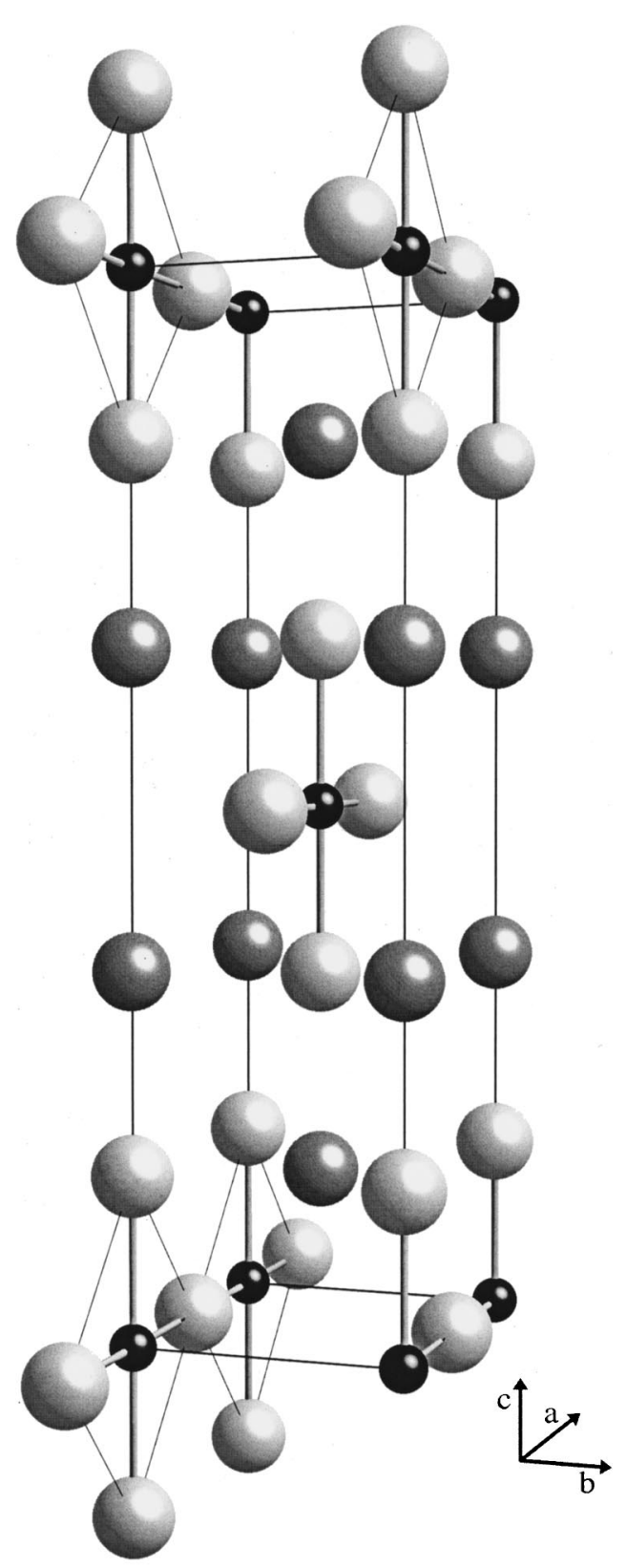

FIG. 1. Crystal structure of $\mathrm{Ca}_{2} \mathrm{CuO}_{3}$. Black spheres represent $\mathrm{Cu}^{2+}$ ions, light gray spheres $\mathrm{O}^{2-}$, and dark gray spheres $\mathrm{Ca}^{2+}$. For some of the copper ions the full oxygen coordination is shown.

\section{METHODS}

\section{A. Pure spin eigenfunctions cluster model calculations}

The crystal structure of $\mathrm{Sr}_{2} \mathrm{CuO}_{3}$ and $\mathrm{Ca}_{2} \mathrm{CuO}_{3}$ is closely related to the perovskite-like structure of $\mathrm{La}_{2} \mathrm{CuO}_{4}$ except for the fact that the copper cations are connected by oxygens along one direction only. This structural difference has, however, important implications. Instead of the characteristic two-dimensional $\mathrm{CuO}_{2}$ planes, spin-1/2 chains are formed that present a variety of particular properties as pointed out in Sec. I. Figure 1 depicts the unit cell and in addition the full oxygen coordination for some of the copper ions is shown to illustrate the formation of the spin chains by corner-sharing $\mathrm{CuO}_{4}$ squares. The space group of both compounds is
$\mathrm{I} / \mathrm{mmm}$ and the lattice parameters are taken from Refs. 50 and 5 for $\mathrm{Ca}_{2} \mathrm{CuO}_{3}$ and $\mathrm{Sr}_{2} \mathrm{CuO}_{3}$, respectively.

A first requirement to obtain accurate $a b$ initio estimates of the different magnetic coupling and effective hopping parameters from a cluster model approach is that the material model applied properly represents the real crystal for the property of interest. A well-established strategy is to divide the system into three regions and to describe each region at a different level of approximation. ${ }^{37-39,48,49,51-56}$ The first region contains two magnetic centers and the oxygen atoms coordinating them. These atoms form the cluster model and are treated at an all-electron level with standard quantum chemical techniques as described afterwards. In the present case, the cluster models are $\mathrm{Cu}_{2} \mathrm{O}_{7}$ for the in-chain interaction (see Fig. 1, basal plane of the unit cell) and $\mathrm{Cu}_{2} \mathrm{O}_{8}$ for the interchain interaction (see Fig. 1, upper plane of the unit cell). The second region accounts for the short-range repulsion between the cluster atoms of region 1 and their near neighbors. For this purpose the centers in region 2 are represented by total ion potentials (TIP's), which prevent the artificial polarization of the charge distribution of the cluster atoms. ${ }^{57}$ The $\mathrm{Cu}_{2} \mathrm{O}_{7}$ cluster is embedded in $2 \mathrm{Cu}^{2+}$ and 16 $\mathrm{X}^{2+}$ TIP's and the $\mathrm{Cu}_{2} \mathrm{O}_{8}$ cluster in $4 \mathrm{Cu}^{2+}$ and $16 \mathrm{X}^{2+}$ TIP's $(\mathrm{X}=\mathrm{Ca}$ or $\mathrm{Sr}$ ). Finally, the third region further surrounds the embedded clusters with a set of optimized point charges that reproduce the Madelung potential with an accuracy better than $0.1 \mathrm{meV}$ in all centers of regions 1 and 2 .

A second, equally important requirement is a correct description of the $\mathrm{N}$-electron wave function to account for the large electron correlation effects, which strongly dominate the properties of interest in the compounds under study. State-of-the-art quantum chemical computational schemes provide a solid base to approximate the eigenfunctions of the exact (nonrelativistic) Hamiltonian of the material model. The simplest approximation follows the description of the superexchange mechanism discussed by Anderson and Nesbet in the late 1950's. ${ }^{58-60}$ It consists in choosing the cluster wave function as a complete active space configuration interaction (CASCI) in the space spanned by the configurations constructed by distributing in all possible ways the unpaired electrons over the open-shell orbitals centered on the magnetic centers. CASCI has included the direct exchangetwice the exchange integral $\left(K_{\mathrm{ab}}\right)$ involving the magnetic orbitals in a localized description-and Anderson delocalization and is known to give a reliable prediction of the sign of the interaction, but it fails to give a quantitative description of the magnitude of the interaction. ${ }^{34-36,48,52,53,61}$

Various approaches exist to construct more sophisticated wave functions that largely improve the description provided by the Anderson model. Here, we apply difference dedicated $\mathrm{CI}^{62}$ in its formulation with two degrees of freedom (DDCI2) or three degrees of freedom (DDCI3). Malrieu showed that up to second-order perturbation theory only those determinants external to the CASCI with two degrees of freedom in the inactive or virtual orbitals contribute to the singlet-triplet splitting. ${ }^{63}$ All other determinants give equal contributions to the energy of both spin states. DDCI2 adds the ligand-tometal CT configurations to the reference CASCI wave function and in addition all other second-order terms that contrib- 
ute to $J$, i.e., dynamic spin polarization, kinetic exchange, etc. as discussed by de Loth and co-workers. ${ }^{64}$ Although DDCI2 largely improves $J$ compared to CASCI, it tends to predict too small absolute values. This indicates that the second-order selection criterion proposed by Malrieu ${ }^{63}$ is certainly a good approximation but needs to be improved and the list of determinants treated in the CI should be extended. The DDCI3 computational scheme has been proven recently to be an excellent tool to derive magnetic coupling parameters in a large family of ionic insulators in very good agreement with experimental data. ${ }^{35,38,65}$ The main feature of the DDCI3 method is that it adds precisely those determinants to the DDCI2 list that cause an energy lowering of the configurations connected with $\mathrm{CT}$ excitations from the bridging ligand to the copper cations. In other words, DDCI3 repairs the large overestimation of $U$ (the effective on-site repulsion parameter in the Hubbard model Hamiltonian) found in Hartree-Fock calculations. The list of determinants treated by DDCI 3 includes much more determinants, but the detailed analysis of Calzado, Sanz, and Malrieu ${ }^{65}$ has established beyond doubt that the only important contribution arises from the determinants that cause the relaxation of the CT excitations. The use of a CI list larger than strictly necessary stems from technical reasons only.

The set of molecular orbitals used in the CI calculations to estimate $t$ and $J$ are those obtained at the Hartree-Fock level for the triplet state. The dependence of $t$ and $J$ on the molecular orbital choice has been discussed by Calzado and co-workers. ${ }^{65}$ They compare the values of $t$ and $J$ for $\mathrm{La}_{2} \mathrm{CuO}_{4}$ calculated with the conventional DDCI applied here and those obtained in the iterative DDCI (IDDCI) approach proposed by García et al. ${ }^{66}$ In the latter approach the orbitals are determined by diagonalizing the density matrix averaged for the two states involved after each CI iteration. This procedure generates an optimal set of molecular orbitals for the property of interest, but is more costly than the conventional DDCI. Although the orbital set (especially in the doped case) differs from the starting set, the results of the IDDCI do not show important changes in the final estimates of $t$ and $J$. This weak dependence on the initial molecular orbitals set can be explained by the fact that the orbital rotations and the optimization of the CI coefficients are connected by a unitary transformation. In other words, the important CT effects in the doped case can show up in the CI expansion (the conventional DDCI case) or in the orbitals (the IDDCI case).

The cluster orbitals are constructed from linear combinations of atomic natural orbitals (ANO's) Gaussian type functions. The contraction scheme used to construct the ANO basis set is specially designed to accurately describe electron correlation effects, yet are as compact as possible. ${ }^{67-69}$ The primitive basis set for $\mathrm{Cu}(21 s, 15 p, 10 d)$ is contracted to $[5 s, 4 p, 3 d]$, for the bridging oxygen $(14 s, 9 p, 4 d) /$ $[4 s, 3 p, 1 d]$ and the edge oxygens $(10 s, 6 p) /[3 s, 2 p]$. Previous applications have shown that a further enlargement of these one-electron basis sets does not significantly affect the values calculated either for $t$ or $J{ }^{48,52,53,70}$

Having established the material model, the $N$-electron wave functions and the atomic basis sets remains to define the way to derive the magnetic coupling parameters $J$ and the effective hopping parameters $t$. To distinguish between the different parameters, we adopt the notation introduced by Rosner et al. ${ }^{13}$ for the $t^{\prime}$ 's and $J$ 's. For the in-chain interactions we use $J_{1}$ for the nearest neighbor and $J_{2}$ for the nextnearest neighbor interaction. The interchain interaction is denoted by $J_{\perp}$. The same notation applies to the $t$ 's. For a comprehensive description of the different mapping procedures we refer to previous work $;^{30,48,65}$ here we only give the basic ideas behind these procedures. $J_{1}$ and $J_{\perp}$ can be obtained from the difference of the energy eigenvalues of the singlet and triplet $\mathrm{N}$-electron eigenfunctions of the total cluster Hamiltonian because these eigenvalues are related in a one-to-one mapping with the eigenvalues of the Heisenberg Hamiltonian for two $S=1 / 2$ centers, i.e., $E_{S}-E_{T}=J$ with $E_{S}$ and $E_{T}$ the energy eigenvalue of the singlet and triplet state, respectively. To derive $J_{2}$ a three center cluster is constructed for which the Heisenberg Hamiltonian reads $\hat{H}=$ $-J_{1}\left(\hat{S}_{1} \hat{S}_{2}+\hat{S}_{2} \hat{S}_{3}\right)-J_{2} \hat{S}_{1} \hat{S}_{3}$. The three $S=1 / 2$ centers in this cluster give rise to a quartet and two different doublet electronic states. The energy eigenvalues are related to $J_{1}$ and $J_{2}$ by the following relations: $J_{1}=2 / 3\left(E_{D_{1}}-E_{Q}\right)$; and $J_{2}=J_{1}-\left(E_{D_{1}}-E_{D_{2}}\right)$, with $E_{D_{1}}, E_{D_{2}}$, and $E_{Q}$ the energy eigenvalues of the two doublets and quartet states, respectively. Calzado and co-workers have shown that the effective hopping parameter $t$ can be written as half the energy difference of the $\mathrm{N}$-electron states in which either the bonding or antibonding combination of the magnetic orbitals is singly occupied.$^{65,70,71}$ Hence, to calculate the hopping parameters, we use a doped cluster model from which one electron has been removed compared to the undoped cluster used to calculate $J$ 's.

All calculations up to the transformation to molecular two-electron integrals have been performed with MOLCAS $4,{ }^{72}$ and the subsequent CI calculations with the CASDI suite of programs. ${ }^{73}$

\section{B. Spin-polarized cluster model calculations}

In the context of solid-state physics, the spin unrestricted also termed spin-polarized methods provide the only possible approach to determine the microscopic parameters $t$ and $J$. Therefore, it is important to assess its performance because these calculations often supply the input for a model Hamiltonian to describe long-range properties. The spin polarized methods can be equally well applied within the cluster model approach, and hence, the comparison between the results obtained by these approaches and those obtained using the quantum chemical methods described in the previous subsection, both within the cluster model approach, permit us to explore the performance of the former in a direct way. On the other hand, the comparison between cluster and periodic calculations within a given spin polarized approach permits us to establish the validity of the cluster model.

The monodeterminantal nature of the spin polarized methods makes it impossible to construct spin functions that are eigenfunctions of $\hat{S}^{2}$ and of the Heisenberg Hamiltonian. Instead, we must describe the different spin arrangements by a 
single Slater of Kohn-Sham determinant that is only an eigenfunction of $\hat{S}_{z}$. Within the cluster model approach, the ferromagnetic state corresponds to a large extent with the maximum $m_{s}$ component of the real triplet function but the antiferromagnetic spin setting mixes the $m_{s}=0$ components of the singlet and triplet functions at equal parts. To obtain estimates of $J$, these functions must be mapped onto the eigenfunctions of the Ising Hamiltonian. Based on the work of Noodleman and Davidson, ${ }^{74}$ Caballol et al. ${ }^{75}$ have shown that the energy difference of the ferromagnetic and antiferromagnetic spin settings equals $1 / 2 J$. For the periodic representation of the materials, similar remarks can be made. The ferromagnetic phase corresponds to the ground state of the Heisenberg Hamiltonian with $J>0$, but the antiferromagnetic alignment, the so-called Néel state, is not an eignestate of the Heisenberg Hamiltonian. Indeed, any interchange of spins on neighboring sites introduces off-diagonal elements in the Heisenberg Hamiltonian that lower the energy with respect to the Néel state. ${ }^{76}$

In the analysis of the performance of the unrestricted methods, cluster models are constructed following the procedure sketched in Sec. II A. One-electron basis sets of similar quality as those in the DDCI calculations are used, to say the $6-3111+\mathrm{G}$ basis set is used for $\mathrm{Cu}$ and the $6-31 G^{*}$ basis set for all oxygen ions in the cluster. Beside the wave function based UHF approach, we also investigate a variety of exchange-correlation functionals. In the first place we calculate $t$ and $J$ values with the LDA functional and with a gradient corrected functional, the recently proposed modified Perdew-Wang functional $(m \mathrm{PW}){ }^{77}$ In contrast to many other gradient corrected functionals, $m \mathrm{PW}$ satisfies a set of important physical conditions that must be fulfilled by the exact functional. The hybrid functionals provide a way to interpolate between the wave function based methods and DFT based methods. These functionals introduce an arbitrary amount of nonlocal Fock exchange in the energy expression. We apply the B3LYP and the B-F:LYP functionals, ${ }^{29,30,47}$ which both use the Lee-Yang-Parr gradient corrected functional $^{78}$ for the correlation part but combine the HF exchange and the gradient corrected exchange functional of Becke differently. B3LYP uses approximately $20 \%$ HF exchange, while B-F:LYP uses a $50 \%$ mixture.

The cluster model UHF and DFT calculations are performed with the GAUSSIAN 98 program. ${ }^{79}$

\section{Periodic calculations}

The periodic calculations are performed with the CRYSTAL98 code ${ }^{80}$ using the unrestricted or spin-polarized approach to account for the magnetic character of these system. ${ }^{81}$ The crystalline orbitals are expressed as linear combinations of Bloch functions, which themselves are constructed from an atomic basis set optimized for the crystal environment. ${ }^{82}$ The atomic basis functions are Gaussian type orbitals of similar quality as applied in the cluster calculations. To extract the magnetic coupling parameters, four different magnetic unit cells with ferromagnetic and antiferromagnetic spin settings are considered. The single unit cell allows us to determine the spin coupling along the body
TABLE I. Magnetic coupling parameters (in meV) for $\mathrm{Ca}_{2} \mathrm{CuO}_{3}$ and $\mathrm{Sr}_{2} \mathrm{CuO}_{3}$ from periodic unrestricted Hartree-Fock calculations.

\begin{tabular}{lcccc}
\hline \hline & $J_{1}\left(=J_{a}\right)$ & $J_{\perp}\left(=J_{b}\right)$ & $J_{c}$ & $J_{d}$ \\
\hline $\mathrm{Ca}_{2} \mathrm{CuO}_{3}$ & -39.8 & -0.41 & 0.00 & -0.01 \\
$\mathrm{Sr}_{2} \mathrm{CuO}_{3}$ & -36.7 & -0.16 & 0.00 & -0.01 \\
\hline \hline
\end{tabular}

diagonal of the unit cell $J_{d}$, and doubling the unit cell along the three crystal axis provides a way to determine the other three spin couplings along the crystal axes $\left(J_{a}=J_{1}, J_{b}\right.$ $=J_{\perp}$, and $J_{c}$ ). The energy differences between the magnetic phases are mapped onto the Ising Hamiltonian to estimate the coupling parameters (cf. Refs. 49 and 56). The values employed to factorize the one-electron equations, the number of $k$ points, and the Coulomb and exchange series ${ }^{45,80}$ are taken from calculations on similar compounds ${ }^{83-85}$ to ensure a correct evaluation of the small energy differences involved in the extraction of the magnetic coupling parameters.

Apart from the mean-field description provided by the UHF scheme, which is known to give too large a band gap, we also study the inclusion of the electron correlation by means of periodic DFT calculations implemented in the CRYSTAL98 code $^{86}$ The auxiliary basis sets for the fitting of the exchange-correlation potential are taken from Ref. 82 .

\section{RESULTS}

\section{A. Magnetic coupling constants}

A simple look at the crystal structure of the two spin chain compounds (Fig. 1) strongly suggests that the only magnetic interaction of importance is that between copper ions along the $a$ axis, where linear $\mathrm{Cu}-\mathrm{O}-\mathrm{Cu}$ bonds give rise to large antiferromagnetic interactions. In addition, it can be anticipated that the copper ions along the $b$ axis have a small interaction, but the interactions along the body diagonal and along the $c$ axis are expected to be extremely small and most probably negligible. Periodic UHF calculations provide a direct and relatively simple way to explore the relative importance of the different magnetic interactions. The results reported in Table I indeed illustrate that only $J_{1}$ and $J_{\perp}$ are of importance. Superexchange interaction along the $c$ axis and the body diagonal $\left(J_{c}\right.$ and $\left.J_{d}\right)$ can be considered to be negligibly small, and a reasonable approximation for these interactions seems to be a classical magnetic dipolar interaction only as adopted by Ami et al. to estimate $J_{\perp}$ [Eq. (6) of Ref. 5].

A second, even more important conclusion can be drawn from these periodic calculations by comparing them to UHF results obtained within the cluster model approach. This comparison provides a rigorous test of the applicability of the cluster model to extract magnetic coupling parameters. The cluster model results listed in Table IV (to which we will come back later on) shows that both $J_{1}$ and $J_{\perp}$ compare very well with the periodic results. The close resemblance between the results obtained by the two approaches shows that, as far as the magnetic coupling is concerned, modeling the three dimensional ionic crystal with a properly embedded 
TABLE II. In-chain and interchain magnetic coupling constants $J_{1}$ and $J_{\perp}$ (in meV) for $\mathrm{Ca}_{2} \mathrm{CuO}_{3}$ and $\mathrm{Sr}_{2} \mathrm{CuO}_{3}$ obtained within the cluster model approach with different pure spin $\mathrm{N}$-electron wave functions.

\begin{tabular}{lccccc}
\hline \hline & & Direct exchange $^{\mathrm{a}}$ & CASCI & DDCI2 & DDCI3 \\
\hline $\mathrm{Ca}_{2} \mathrm{CuO}_{3}$ & $J_{1}$ & 10.63 & -34.7 & -124 & -231 \\
& $J_{\perp}$ & 0.02 & -0.03 & -0.72 & -0.72 \\
& & 10.81 & -34.8 & -130 & -246 \\
$\mathrm{Sr}_{2} \mathrm{CuO}_{3}$ & $J_{1}$ & 0.02 & -0.01 & -0.37 & -0.44 \\
& $J_{\perp}$ & &
\end{tabular}

${ }^{\text {a }}$ Two times the exchange integral $K_{a b}$, where $a$ and $b$ refer to the localized magnetic orbitals.

cluster containing only a few atoms does not introduce serious artifacts in the calculation of the magnetic interaction parameter. This observation is not unique for $\mathrm{Sr}_{2} \mathrm{CuO}_{3}$ and $\mathrm{Ca}_{2} \mathrm{CuO}_{3}$, but has been reported before for other ionic insulators as $\mathrm{KNiF}_{3}, \mathrm{~K}_{2} \mathrm{NiF}_{4}, \mathrm{KCuF}_{3}, \mathrm{La}_{2} \mathrm{CuO}_{4}$, and $\mathrm{CuF}_{2} \cdot{ }^{44,48,49,55,83}$ More evidence for the reliability of the cluster model approach has been given by Illas and co-workers ${ }^{54}$ in a study of the dependence of $J$ upon the number of magnetic centers explicitly included in the cluster model. The magnetic interactions calculated in a two-center cluster were found to be virtually the same as those in a three-center or four-center cluster.

The large advantage of the cluster model approach above periodic calculations is that electron correlation effects can be introduced in a straightforward and systematic manner. Table II reports the in-chain and interchain magnetic coupling parameters at four different levels of approximation of the $N$-electron wave function. In the first place, we notice that as expected the direct exchange is always ferromagnetic. While it has a significant contribution for the in-chain interaction, it is almost zero for the interchain interaction. This is consistent with the fact that the magnetic orbitals $\left(\mathrm{Cu} 3 d_{x^{2}-y^{2}}\right)$ do not have significant density along the $z$ axis, the interchain direction. On the other hand, CASCI properly predicts both $J_{1}$ and $J_{\perp}$ to be antiferromagnetic, but the absolute values are much smaller than any of the values given in the literature.

A more realistic description is reached by the DDCI2 method; $J_{1}$ is enhanced by a factor of approximately 3.5 and $J_{\perp}$ is now clearly antiferromagnetic as predicted by UHF cluster (vide infra) and periodic calculations. Although the inclusion of the second-order terms described in Sec. II largely improves the description of the magnetic coupling process, an important term is still missing and-as previous work shows - a quantitative description is only obtained by activating the instantaneous relaxation of the ligand-to-metal CT excitations. The DDCI3 values listed in Table II have included this effect and give our final and most reliable $a b$ initio estimates of $J_{1}$ and $J_{\perp}$ for $\mathrm{Ca}_{2} \mathrm{CuO}_{3}$ and $\mathrm{Sr}_{2} \mathrm{CuO}_{3}$. In the first place, we note that DDCI3 increases the in-chain interaction by a factor of 2 for both compounds, whereas the interchain interaction is hardly affected by the extension of the CI space. A look at the structure provides a simple explanation for this difference. The in-chain interaction takes place along linear $\mathrm{Cu}-\mathrm{O}-\mathrm{Cu}$ bonds, for which significant $\mathrm{O} 2 p$ to $\mathrm{Cu} 3 d \mathrm{CT}$ excitations can be expected. On the other hand, the lack of a bridging ligand along the $b$ axis makes the interchain interaction basically a $\mathrm{Cu}-\mathrm{Cu}$ exchange interaction without CT contributions, and hence it is not at all unexpected that DDCI3 does not add any new physics to the DDCI2 wave function.

Second, it is interesting to see that $J_{1}$ is slightly smaller in $\mathrm{Ca}_{2} \mathrm{CuO}_{3}$, although the $\mathrm{Cu}-\mathrm{O}-\mathrm{Cu}$ bond is smaller compared to $\mathrm{Sr}_{2} \mathrm{CuO}_{3}$ (3.79 versus $3.91 \AA$ ) and from this point of view a smaller interaction is expected. The interchain interaction, on the other hand, is in line with the $\mathrm{Cu}-\mathrm{Cu}$ distances in the two compounds (3.28 versus $3.49 \AA$ ). The larger interchain distance in $\mathrm{Sr}_{2} \mathrm{CuO}_{3}$ leads to a smaller magnetic coupling. The origin of this apparent contradiction can be elucidated by means of a computational experiment consisting in the calculation of $J_{1}$ and $J_{\perp}$ for $\mathrm{Ca}_{2} \mathrm{CuO}_{3}$, but applying the lattice parameters of $\mathrm{Sr}_{2} \mathrm{CuO}_{3}$. Note that this fictitious system only differs from real $\mathrm{Sr}_{2} \mathrm{CuO}_{3}$ in the representation of the 16 counterions directly surrounding the $\mathrm{Cu}_{2} \mathrm{O}_{7}$ cluster, $\mathrm{Ca}^{2+}$ TIP's instead of $\mathrm{Sr}^{2+}$ TIP's and in the Madelung potential provided by the array of point charges. DDCI2 predicts a rather large effect due to the larger lattice parameters: $J_{1}$ becomes $-104 \mathrm{meV}$ (we skip the DDCI3 calculation, being rather expensive computationally, while DDCI2 very well reproduces trends) and $J_{\perp}$ equals $-0.44 \mathrm{meV}$. This indicates that the larger size of the $\mathrm{Sr}^{2+}$ ions counterbalances the $\mathrm{Cu}$ $\mathrm{O}-\mathrm{Cu}$ bond elongation going from $\mathrm{Ca}_{2} \mathrm{CuO}_{3}$ to $\mathrm{Sr}_{2} \mathrm{CuO}_{3}$. On the contrary, since the direct exchange contribution to the interchain interaction is negligible (cf. Table II), it turns out that this magnetic coupling is completely determined by the $\mathrm{Cu}-\mathrm{Cu}$ distances and largely independent of the size of the counterions.

Finally, we compare the DDCI3 results with data listed in Sec. I. Our results are in excellent agreement with the interpretation by Lorenzana and Eder of the phonon-assisted magnetic excitations ${ }^{12}$ and the NMR data reported by Takigawa et al. ${ }^{11,22}$ and confirm $\mathrm{Ca}_{2} \mathrm{CuO}_{3}$ and $\mathrm{Sr}_{2} \mathrm{CuO}_{3}$ to have exchange integrals that are much larger than in any other cuprate. These large magnetic couplings are incompatible with the analysis of the magnetic susceptibility data ${ }^{18,19}$ based on the accurate expression of Eggert, Affleck, and Takahashi $^{9}$ and the rough estimate derived from ARPES. ${ }^{20}$ At this point a comparison must also be made with another cluster model study of the magnetic interactions ${ }^{87}$ based on the nonorthogonal CI approach (NOCI). ${ }^{88}$ This computational scheme gives magnetic coupling parameters in a series of undoped cuprates: $\mathrm{La}_{2} \mathrm{CuO}_{4}, \mathrm{Nd}_{2} \mathrm{CuO}_{4}, \mathrm{YBa}_{2} \mathrm{Cu}_{3} \mathrm{O}_{6}$, and $\mathrm{Sr}_{2} \mathrm{CuO}_{2} \mathrm{Cl}_{2},{ }^{34}$ in rather good agreement with experimental data and comparable to DDCI3 values, ${ }^{38}$ although NOCI values are always somewhat smaller than those obtained with DDCI3. Nevertheless, in the present case a NOCI study predicts $J_{1}$ to be $136 \mathrm{meV}$ both for $\mathrm{Ca}_{2} \mathrm{CuO}_{3}$ and $\mathrm{Sr}_{2} \mathrm{CuO}_{3}$, in obvious contradiction to the DDCI3 values reported in Table II. Two reasons can be given to explain the difference between the two computational approaches. In the first place, the embedding used in the NOCI calculations is less precise and has a more ad hoc character than the well-defined TIPs we use in the DDCI3 calculations. Another factor to be con- 
sidered is that apart from the relaxed CT excitations, the NOCI study did not include any of the other mechanisms accounted for in DDCI2 and/or DDCI3. Hence, we conclude that the computational scheme adopted in the present work is more precise and gives a more complete description of the magnetic coupling.

The next-nearest neighbor in-chain interaction $J_{2}$ is also accessible in the cluster model approach by extending the cluster with a third $\mathrm{Cu}^{2+}$ ion and its neighboring oxygen ions. Nevertheless, the computational effort becomes much larger now, and therefore, we estimate this parameter by complete active space second-order perturbation theory $(\text { CASPT2 })^{89,90}$ instead of the variational DDCI method. Although this more approximate method does not quantitatively reproduce the magnetic coupling strength, it does very accurately predict trends and relative magnitudes, and hence is very suitable to give an estimate of the relative importance of $J_{2}$ compared to $J_{1}$. For $\mathrm{Ca}_{2} \mathrm{CuO}_{3}, J_{2}$ turns out to be weakly ferromagnetic, $5.5 \mathrm{meV}$, to be compared with the $-162 \mathrm{meV}$ calculated for $J_{1}$ with the same method in the same three-center cluster. This means that the next-nearest neighbor interaction is about $3.5 \%$ of the nearest neighbor interaction, and consequently, the extrapolation of this percentage to the final DDCI 3 value of $J_{1}$ can give us a rough first estimate of the size of $J_{2}$, being $+7.8 \mathrm{meV}$. The results for $\mathrm{Sr}_{2} \mathrm{CuO}_{3}$ are very similar: at the CASPT2 level $J_{2}$ is $+5.9 \mathrm{meV}$ versus $-171 \mathrm{meV}$ for $J_{1}$, again $\sim 3.5 \%$ of $J_{1}$. Hence, the extrapolation to DDCI3 results in an estimate for $J_{2}$ of $8.6 \mathrm{meV}$ in $\mathrm{Sr}_{2} \mathrm{CuO}_{3}$. The three-center calculations permit us to repeat the check on the cluster size convergence discussed in previous work $^{54}$ by comparing them with equivalent calculations in the two-center model. CASPT2 values for $J_{1}$ in the two-center cluster are $-168 \mathrm{meV}$ for $\mathrm{Ca}_{2} \mathrm{CuO}_{3}$ and $-177 \mathrm{meV}$ for $\mathrm{Sr}_{2} \mathrm{CuO}_{3}$. As expected, these values closely resemble those derived from the three-center cluster.

\section{B. Hopping parameters}

The $t-J$ model Hamiltonian defines a simple yet rather accurate model to study the low-energy properties of the $\mathrm{CuO}_{2}$ planes in doped copper oxides. ${ }^{91}$ The model is easily extended to a $t-t^{\prime}-J$ model (or similar variants) to incorporate mechanisms other than those occurring between nearest neighbors in the $\mathrm{CuO}_{2}$ planes. The model is also useful to study other systems for which the crystal structure strongly suggests that the low-energy physics cannot be described with just one $t$ and one $J$. In the spin compounds, it is clear that at least the interchain magnetic coupling and the hopping parameters must be added to the Hamiltonian. In addition, Rosner et al. ${ }^{13}$ suggest that hopping processes to second neighbors are also important. In order to complete the list of ab initio parameters that can serve as input to construct an effective Hamiltonian such as the extended $t-J$ model, we present in this section calculations aimed at an accurate estimation of the hopping parameters. Indeed, this is of crucial importance because direct experimental estimates of these parameters are generally not available.
TABLE III. In-chain and interchain effective hopping parameters $t_{1}$ and $t_{\perp}$ (in meV) for $\mathrm{Ca}_{2} \mathrm{CuO}_{3}$ and $\mathrm{Sr}_{2} \mathrm{CuO}_{3}$ obtained within the cluster model approach with different pure spin $\mathrm{N}$-electron wave functions.

\begin{tabular}{lcccc}
\hline \hline & & CASCI & DDCI2 & DDCI3 \\
\hline $\mathrm{Ca}_{2} \mathrm{CuO}_{3}$ & $t_{1}$ & 639 & 551 & 653 \\
& $t_{\perp}$ & 21.0 & 29.9 & 40.4 \\
$\mathrm{Sr}_{2} \mathrm{CuO}_{3}$ & $t_{1}$ & 639 & 552 & 659 \\
& $t_{\perp}$ & 16.9 & 22.0 & 30.2 \\
\hline \hline
\end{tabular}

The ability of the cluster model approach to calculate accurate estimates of the hopping parameters has been demonstrated by Calzado et al. ${ }^{65,70}$ in their study of $t$ in $\mathrm{La}_{2-x} \mathrm{Sr}_{x} \mathrm{CuO}_{4}$. In Sec. IIIC we give additional evidence in favor of the cluster model approach by comparing with periodic calculations. Table III reports the in-chain and interchain hopping parameters for both compounds at different levels of approximation of the $N$-electron wave function. A first striking observation is the fact that both $t_{1}$ and $t_{\perp}$ show a much weaker variation with the different computational schemes. Second, it is recognized that while $t_{1}$ is relatively similar in $\mathrm{Ca}_{2} \mathrm{CuO}_{3}$ and $\mathrm{Sr}_{2} \mathrm{CuO}_{3}, t_{\perp}$ is $50 \%$ larger in $\mathrm{Ca}_{2} \mathrm{CuO}_{3}$ in agreement with the shorter interchain distance for this compound. The absolute values of the hopping parameters are in fair agreement with the LDA values reported by Rosner et al. ${ }^{13}$ but the more elaborate treatment of the electron correlation effects makes the DDCI3 result a more reliable estimate of the magnitude hopping integral. Moreover, it is important to point out the fact that LDA and DDCI3 both agree in the estimate of $t$ (vide infra) while largely differing in the predictions of $J$, the former most often being completely unable to produce even qualitatively correct results.

Another matter of concern is the character of the holes in the doped material. This character can be assessed by comparing the contributions to the DDCI3 wave function of CT configurations with the hole localized on the oxygen ions versus the contributions of the non-CT configurations that represent the unscreened hole. This analysis shows that the hole has approximately a $50 \% \mathrm{O} 2 p$ character in agreement with the general understanding behind the single band $t-J$ model of Zhang and Rice, and also previously found in the study of the hopping processes in $\mathrm{La}_{2-x} \mathrm{Sr}_{x} \mathrm{CuO}_{4}{ }^{65} \mathrm{~A}$ similar analysis for the undoped system reveals a contribution as high as $90 \%$ of the non-CT configurations to the DDCI3 wave function. Since the importance of the CT determinants in the doped system is about as important as the non-CT determinants, it can be argued that these determinants must be included in the reference wave function. However, the variational determination of the DDCI3 wave function ensures a treatment on equal footing with the $\mathrm{CT}$ and non-CT determinants. Therefore, there is no need to include the CT determinants in the reference space or to change the molecular orbital set used in the CI. Another concern is the different quality of the bridging oxygen and the edge oxygens, although the role of all oxygens is expected to be of similar 
TABLE IV. In-chain and interchain magnetic coupling constants $J_{1}$ and $J_{\perp}$ (in meV) for $\mathrm{Ca}_{2} \mathrm{CuO}_{3}$ and $\mathrm{Sr}_{2} \mathrm{CuO}_{3}$ obtained within the cluster model approach with different spin unrestricted methods.

\begin{tabular}{lcccccc}
\hline \hline & & UHF & B-F:LYP & B3LYP & $m$ PW & LDA \\
\hline $\mathrm{Ca}_{2} \mathrm{CuO}_{3}$ & $J_{1}$ & -45.7 & -142 & -339 & -839 & -1105 \\
& $J_{\perp}$ & -0.91 & -1.75 & -1.96 & -2.29 & -2.75 \\
$\mathrm{Sr}_{2} \mathrm{CuO}_{3}$ & $J_{1}$ & -43.7 & -144 & -355 & -879 & -1151 \\
& $J_{\perp}$ & -0.42 & -0.93 & -1.01 & -1.01 & -1.15 \\
\hline \hline
\end{tabular}

importance in the description of the hole states. However, by repeating the calculations for $t_{1}$ of $\mathrm{Ca}_{2} \mathrm{CuO}_{3}$ reported in Table III with a better balanced basis set- $(4 s, 3 p)$ for all oxygens-we find that the description of the hole state is not largely affected by this slight unbalance in the one-electron basis set. The values of $t_{1}$ with the alternative basis set are $624 \mathrm{meV}$ for CASCI, $544 \mathrm{meV}$ for DDCI2, and $632 \mathrm{meV}$ for DDCI3.

Finally, we note that the next-nearest neighbor hopping integral $t_{2}$ cannot be calculated as straightforward as the other parameters discussed so far. This is because $t_{2}$ is not simply related to an energy difference between two electronic states. In fact it has been proven that it is indispensable to construct a $(6 \times 6)$ effective Hamiltonian to derive this parameter. ${ }^{92}$ Work is in progress to calculate $a b$ initio estimates for this parameter too.

\section{Magnetic coupling and hopping parameters from spin-polarized methods}

As stated in Sec. I, LDA badly fails to reproduce the insulating character of $\mathrm{Ca}_{2} \mathrm{CuO}_{3}$ and $\mathrm{Sr}_{2} \mathrm{CuO}_{3}$ and other ionic transition metal compounds. Nevertheless, in many applications model parameters have been derived from LDA calculations (see for example Refs. 13, 28, and 93-95) to interpret experimental data. Therefore, it is interesting to see to what extent LDA and other spin-polarized methods are capable of properly predicting the magnetic coupling and hopping parameters of the compounds under study. Table IV compares cluster model results for $J_{1}$ and $J_{\perp}$ obtained with UHF, LDA, a gradient corrected functional ( $m \mathrm{PW})$, and two hybrid functionals (B-F:LYP and B3LYP). Table V lists the same data for $t_{1}$ and $t_{\perp}$. It is worth pointing out again the excellent agreement between cluster and periodic UHF values of the magnetic coupling constants. Hence, any defi-

TABLE V. In-chain and interchain effective hopping parameters $t_{1}$ and $t_{\perp}$ (in meV) for $\mathrm{Ca}_{2} \mathrm{CuO}_{3}$ and $\mathrm{Sr}_{2} \mathrm{CuO}_{3}$ obtained within the cluster model approach with different spin unrestricted methods.

\begin{tabular}{lcccccc}
\hline \hline & & UHF & B-F:LYP & B3LYP & $m$ PW & LDA \\
\hline $\mathrm{Ca}_{2} \mathrm{CuO}_{3}$ & $t_{1}$ & 790 & 633 & 541 & 486 & 491 \\
& $t_{\perp}$ & 26.6 & 26.1 & 23.5 & 21.4 & 21.8 \\
$\mathrm{Sr}_{2} \mathrm{CuO}_{3}$ & $t_{1}$ & 795 & 639 & 548 & 488 & 496 \\
& $t_{\perp}$ & 16.8 & 15.7 & 14.0 & 12.6 & 13.0 \\
\hline \hline
\end{tabular}

ciency in the density functional based approaches in the forthcoming analysis cannot be attributed to the use of a cluster model but the particular theoretical approach under discussion.

To start with the in-chain interaction, it can be readily concluded that the tendency of LDA to incorrectly predict insulators as metallic systems result in magnetic interactions along $\mathrm{Cu}-\mathrm{O}-\mathrm{Cu}$ bonds that are unphysically large. In fact, the $\mathrm{AF}$ state corresponds to a closed-shell solution, i.e., the equivalent of a metallic state in a solid-state description. The use of a gradient corrected functional does not largely improve the LDA result. In fact, $J_{1}$ decreases somewhat but is still far outside the experimental range of data. On the other extreme, we find the UHF approach, which predicts too small an interaction. The application of hybrid functionals results in parameters that lie between these two extremes and, depending on the amount of HF exchange, the value closer resembles the LDA or UHF result. Hence, it is in principle possible to construct a hybrid functional that accurately reproduces the DDCI3 values listed in Table II (or experimental data for compounds for which there is a clear consensus about the size of the coupling) by optimizing the amount of HF exchange in the functional. Note, however, that there is no systematic well-grounded way to do this and the resulting method cannot be considered as an $a b$ initio method since it requires information external to theory. Moreover, the optimum amount of HF exchange can differ from compound to compound, e.g., Martin and Illas ${ }^{29,30}$ have shown that F-B:LYP fairly well reproduces the magnetic coupling constant in $\mathrm{La}_{2} \mathrm{CuO}_{4}$, but the present results show that for the spin-chain compounds $\mathrm{Sr}_{2} \mathrm{CuO}_{3}$ and $\mathrm{Ca}_{2} \mathrm{CuO}_{3}$ the amount of $\mathrm{HF}$ exchange has to be significantly smaller in order to obtain accurate values.

The interchain interaction $J_{\perp}$ is less dependent on the computational scheme applied. Since there is no direct trough-bond connection between the two coppers involved in this interaction, the tendency of LDA to overestimate delocalization effects is less pronounced. All methods give values that are relatively close to DDCI3, however the lack of a systematic way to order the different spin-polarized schemes in increasing accuracy makes it difficult to analyze the differences between them. This is in contrast to the spinrestricted schemes discussed in the previous sections, for which a clear-cut analysis can be made of the mechanisms included at each step.

We now turn our attention to the calculation of the hopping parameters within the spin-polarized approach. In contrast to the behavior found for the magnetic coupling parameter, all five methods give hopping parameters of the same order of magnitude not too different from the previously discussed DDCI3 results. In fact, the LDA estimate of $t_{1}$ is only $25 \%$ smaller than the DDCI3 value, whereas a difference of almost $400 \%$ is found for $J_{1}$. Therefore, an important point involves a comparison with the results of the LDA periodic calculations of Rosner et $a l .{ }^{13}$ In this work the hopping parameters are calculated from a fit of the LDA band structure and hence provide us with a way to validate the cluster model approach for the calculation of the hopping parameters similar to the reasoning extensively used for the $J$ 's. 
The values of $t_{1}$ reported by Rosner et al. (520 and $550 \mathrm{meV}$ for $\mathrm{Ca}_{2} \mathrm{CuO}_{3}$ and $\mathrm{Sr}_{2} \mathrm{CuO}_{3}$, respectively) are in good agreement with those in Table V, 491 and $496 \mathrm{meV}$, which are also obtained from LDA calculations but within the cluster model approach. The interchain hopping parameters are also comparable in the cluster model and periodic approaches, although the small value of $t_{\perp}$ makes the relative error larger.

\section{Electronic structure}

The periodic UHF calculations mentioned at the beginning of Sec. III A not only give support to the cluster model approach but also supply important information about the electronic structure of the two compounds. In the first place, we observe that the Mulliken charges are close to the formal ionic values; +1.8 for $\mathrm{Cu},+1.9$ for $\mathrm{Ca}$ and $\mathrm{Sr}$, and -1.9 for O. Furthermore, the population of the $d$ band, 9.13, is very close to the formal value of a $\mathrm{Cu}^{2+}$ ion in vacuum. Without imposing external restrictions, the $\left(x^{2}-y^{2}\right)$ symmetry of the singly occupied $d$ orbital emerges naturally from the optimization of the crystal wave function. The spin density plots clearly illustrate the superexchange mechanism, giving rise to the large antiferromagnetic interactions in the spin chains. Beside the obvious spin density centered on the $\mathrm{Cu}$ ion with $d_{x^{2}-y^{2}}$ symmetry, we observe that the $\alpha$ electrons on the bridging oxygen polarize towards the $\mathrm{Cu}$ ion with $m_{s}=$ $+1 / 2$ and vice versa for the $\beta$ electrons. On the other hand no such mechanism is possible for the interchain interaction. Neither the $\mathrm{Cu}$ nor the oxygen ions show a significant spin polarization along the $b$ axis. Furthermore, these calculations indicate that the spin density on the $\mathrm{Ca}$ (and $\mathrm{Sr}$ ) ions is less than 0.008 and can be considered to play a negligible role in the interchain interaction in agreement with the observations of the cluster calculations.

Although the UHF approach gives a good qualitative picture of many properties of solid state compounds, it notoriously fails to give a quantitative description of the band gap. For both $\mathrm{Sr}_{2} \mathrm{CuO}_{3}$ and $\mathrm{Ca}_{2} \mathrm{CuO}_{3}$, the UHF band gap is as large as $16 \mathrm{eV}$. Hence, serious doubts arise about the applicability of this method to interpret the density of states and to study the character of the band gap. This well-known failure of UHF is due to the neglect of the dynamical electron correlation effects. Since these effects are not easily incorporated in a periodic wave function based approach-only recently a method has been developed to obtain periodic correlated wave functions for ground state properties ${ }^{96}$-we opt for calculations based on the density functional theory applying the B-F:LYP and B3LYP exchange-correlation functionals as explained in Sec. II. The first functional already largely reduces the band gap to $\sim 6 \mathrm{eV}$, but the B3LYP functional gives a band gap that is in reasonable agreement with the experimental estimates, namely $1.53 \mathrm{eV}$ for $\mathrm{Ca}_{2} \mathrm{CuO}_{3}$ and $1.82 \mathrm{eV}$ for $\mathrm{Sr}_{2} \mathrm{CuO}_{3}$. This decrease of the band gap is accompanied by a modest increase of the covalent character of the $\mathrm{Cu}-\mathrm{O}$ bonds. The Mulliken charges resulting from the B3LYP calculation are 1.5 for $\mathrm{Cu}, 1.8$ for $\mathrm{Sr}$, and -1.7 for $\mathrm{O}$. Note that the trend in the band gap is directly related to the trend observed in Sec. III C for the magnetic coupling parameters. LDA predicts that the com-

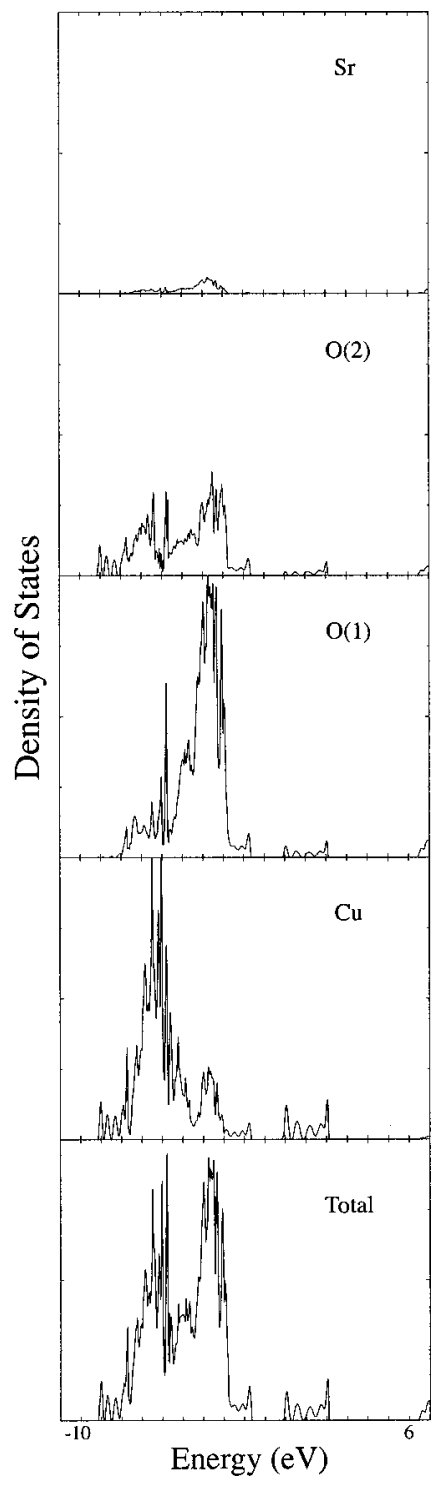

FIG. 2. B3LYP total and partial densities of states of antiferromagnetic $\mathrm{Ca}_{2} \mathrm{CuO}_{3}$. $\mathrm{O}(1)$ refers to the in-chain oxygens and $\mathrm{O}(2)$ to the apex oxygens.

pounds are metallic, which results in a magnetic coupling parameter that is unphysically large, while UHF predicts too large a band gap and gives a $J$ value that is only $15 \%$ of the final theoretical estimate. The hybrid functionals again represent intermediate cases: BFLYP gives a band gap that is somewhat too large and with B3LYP it comes out somewhat too small. Unfortunately, none of the existing functionals seems to be able to simultaneously describe the magnitude of the magnetic coupling and of the band gap.

Figure 2 display the density of states (DOS's) for $\mathrm{Sr}_{2} \mathrm{CuO}_{3}$ calculated with B3LYP and Fig. 3 zooms in on the region around the top of the valence band and the bottom of the conduction band for $\mathrm{Ca}_{2} \mathrm{CuO}_{3}$. The DOS's of both compounds are qualitatively very similar. As was also observed in the LDA band structure, ${ }^{13}$ the band edges show the characteristic van Hove singularities. These singularities play an important role in the explanation of many phenomena related to superconductivity, but are less interesting for 1D materials 


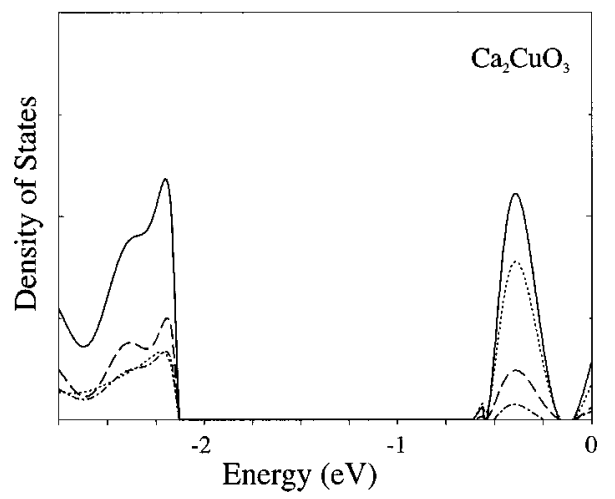

FIG. 3. Enlargement of the B3LYP total and partial densities of states of antiferromagnetic $\mathrm{Ca}_{2} \mathrm{CuO}_{3}$ at the top of the valence band and the bottom of the conduction band. Full lines represent the total density of states, dotted lines the $\mathrm{Cu}$ density, dashed lines the inchain oxygens (O1), and dashed-dotted lines the apex oxygens (O2).

because they appear at the band edge where the number of free carriers is small. ${ }^{97}$ In contrast to LDA, the top of the valence band is well separated from the unoccupied levels but does not appear as an isolated band separated from the broad complex between -9 and $-3 \mathrm{eV}$, which is mainly composed of $\mathrm{Cu} 3 d$ and $\mathrm{O} 2 p$ levels with negligible contributions from the $\mathrm{Ca}$ or $\mathrm{Sr}$ ions. From Fig. 3, a clear analysis of the character of the band gap can be made. The top of the valence band is composed of the $2 p$ levels of both the inchain and the apex oxygens with a significant contribution from the occupied $\mathrm{Cu} 3 d$ levels. The bottom of the conduction band, on the other hand, can be considered to a high extent as a pure $\mathrm{Cu} 3 d$ band, i.e., the upper Hubbard band. Hence, we do not observe any indication of the existence of a so-called covalent correlated insulator as suggested by Maiti et al. ${ }^{25}$ and an interpretation in terms of a CT insulator seems more appropriate.

Finally, we comment on the appearance of unoccupied levels at negative energies, which do not have any physical meaning but are caused by artifacts of the computational scheme. From molecular calculations it is well known that DFT systematically underestimates the highest occupied molecular orbital-lowest unoccupied molecular orbital gap since both occupied and unoccupied levels are optimized in the potential due to the same number of electrons. The $\sim 20 \%$ of Hartree-Fock exchange in the B3LYP functional prevents the unoccupied levels from collapsing into the valence band, as occurs for LDA, but obviously the amount of Hartree-Fock exchange should be somewhat larger to shift all energies to positive energies. Again, the lack of an independent criterion to determine the optimum amount of Hartree-Fock exchange makes this strategy an uninteresting one, as already discussed for the magnetic coupling parameters.

\section{E. Parameter consistency}

In view of the aim of providing reliable quantitative estimates for the magnetic coupling and the hopping parameters in the spin chain compounds, it is an interesting exercise to test these parameters against predictions arising from theo-
TABLE VI. Test on the consistency of the parameters derived from the embedded cluster calculations (see text). The effective on-site repulsion, $U$ derived from $U=4 t_{1}^{2} / J_{1}$ is given in $\mathrm{eV}$.

\begin{tabular}{ccccccc}
\hline \hline & & CASCI & DDCI2 & DDCI3 & UHF & LDA \\
\hline $\mathrm{Ca}_{2} \mathrm{CuO}_{3}$ & $\left(t_{1} / t_{\perp}\right)^{2}$ & 926 & 340 & 261 & 882 & 507 \\
& $J_{1} / J_{\perp}$ & 1157 & 172 & 321 & 50 & 402 \\
& $U$ & 47.1 & 9.8 & 7.3 & 54.6 & 0.87 \\
& & & & & & \\
$\mathrm{Sr}_{2} \mathrm{CuO}_{3}$ & $\left(t_{1} / t_{\perp}\right)^{2}$ & 1430 & 630 & 476 & 2239 & 1456 \\
& $J_{1} / J_{\perp}$ & 2486 & 351 & 559 & 104 & 1001 \\
& $U$ & 46.9 & 9.4 & 7.1 & 57.9 & 0.85 \\
\hline \hline
\end{tabular}

ries external to the method used to derive them. Internal tests such as the dependency on the basis set, on the $N$-electron wave function, or on the cluster size, indicate that the calculated values for $t$ and $J$ are basically converged for these variables. The first external test has already been discussed, namely a comparison of the cluster model approach and the periodic calculations. Both for $J$ and $t$ this comparison is very satisfactory. In this section, the parameters are tested against two other theories. In the first place, we calculate the Néel temperature with the mean-field expression given by Schulz $^{14}$ and second we show that our parameters are consistent with simple relations that emerge naturally from the $t$ - $J$ model.

Following the procedure previously adopted by other authors, ${ }^{13,87}$ we replace the interchain interaction in the expression of Schulz by $0.5\left(J_{\perp}+J_{c}\right)=0.5 J_{\perp}$, since $J_{c}$ can safely be considered to be close to zero (cf. Table I). The substitution of the DDCI3 parameters in the resulting expression gives a Néel temperature of $14.2 \mathrm{~K}$ for $\mathrm{Ca}_{2} \mathrm{CuO}_{3}$ and 9.0 $\mathrm{K}$ for $\mathrm{Sr}_{2} \mathrm{CuO}_{3}$, to be compared with the experimental values of 11 and $5.4 \mathrm{~K}$. Because the mean-field description is known to overestimate the Néel temperature, the DDCI3 estimates of the magnetic coupling parameters can be considered to be consistent with the very low experimental Néel temperature. Moreover, the substitution of the parameters in the expression given by Schulz to estimate the magnetic moments results in values of $0.09 \mu_{B}$ for $\mathrm{Ca}_{2} \mathrm{CuO}_{3}$ and $0.03 \mu_{B}$ for $\mathrm{Sr}_{2} \mathrm{CuO}_{3}$, also in good agreement with the experimental values of 0.09 and $0.06 \mu_{B}$.

The second test not only involves the magnetic coupling parameters but also the hopping parameters and adopts a strategy previously applied to check the coherence of parameters calculated for $\alpha^{\prime}-\mathrm{NaV}_{2} \mathrm{O}_{5} .{ }^{39}$ The perturbation expansion in $t / U$ of the extended Hubbard model leads to a simple and widely applied expression to relate $t$ and $J$ that reads $J$ $=4 t^{2} / U$. This relation directly implies that the ratio $J_{1} / J_{\perp}$ is equal to $\left(t_{1} / t_{\perp}\right)^{2}$. Table VI lists the two ratios for a selection of computational schemes, namely CASCI, DDCI2, DDCI3, and the spin-polarized methods UHF and LDA. In addition, the table also gives an estimate of the on-site repulsion parameter $U$ derived from the relation $U=4 t_{1}^{2} / J_{1}$. Note, however, that this estimate is by no means meant to give either an accurate or an $a b$ initio value of the magnitude of $U$, but only serves to discard sets of parameters that give rise to absurd results. All methods with the exception of UHF give 
ratios that are reasonably similar to each other, although the DDCI3 set of parameters are clearly the most consistent ones with a deviation of the ratios of $19 \%$ for $\mathrm{Ca}_{2} \mathrm{CuO}_{3}$ and $15 \%$ for $\mathrm{Sr}_{2} \mathrm{CuO}_{3}$. CASCI and LDA give similar deviations of $20 \%-40 \%$, and DDCI2 performs a little worse because of the relatively large underestimation of $J_{1}$ in comparison to $J_{\perp}$. However, if we focus on the (rough) estimate of $U$, it is immediately clear that both CASCI and LDA give rise to values that are unrealistic; CASCI largely overestimates $U$, while LDA gives too small a value. The inclusion of electron correlation effects going from CASCI to DDCI2 largely reduces $U$ and the inclusion of the determinants that instantaneously relax the CT configurations by means of DDCI3 gives a further reduction of $U$, bringing it within a range of reasonable values.

In short, we conclude that the set of parameters calculated within the DDCI3 computational scheme gives a consistent set of parameters, both for the Néel temperature and for the ratios between in-chain and interchain parameters and the estimate of $U$.

\section{SUMMARY}

We have performed $a b$ initio cluster calculations to investigate the magnetic interactions and effective hopping parameters in the spin-chain compounds $\mathrm{Ca}_{2} \mathrm{CuO}_{3}$ and $\mathrm{Sr}_{2} \mathrm{CuO}_{3}$. In addition we have studied the electronic structure and character of the band gap of these compounds by means of HartreeFock and/or density functional periodic calculations. The strong point of the cluster calculations is that electron correlation effects can be included in the wave function in a controlled systematic way, in this way improving the approximation to the exact $N$-electron wave function step by step. The weak point of this approach is the representation of a periodic material with a small cluster containing only few atoms, which could be questioned as being sufficient. However, the combination of cluster and periodic calculations applied in the present work allows for a rigorous test of the material model adopted in the cluster model approach. By comparing the results of the two approaches at identical levels of theory, we have shown that the cluster model approach is a valid one to derive $a b$ initio estimates of the parameters of an extended $t-J$ model that includes in-chain and interchain processes.

The magnitude of the magnetic coupling along the $\mathrm{Cu}$ $\mathrm{O}-\mathrm{Cu}$ bonds that form the spin chains is found to be $\sim 240$ $\mathrm{meV}$ for both compounds. This is significantly larger than found in any other cuprate. Our results are in good agreement with the analysis of the phonon-assisted magnetic excitations observed in the midinfrared spectrum of these compounds and ${ }^{63} \mathrm{Cu}$ NMR data, but in obvious contradiction with smaller estimates obtained from fitting of magnetic susceptibility data or ARPES experiments. The interchain magnetic interaction is antiferromagnetic but rather weak as can be expected since there is no ligand connecting the copper ions in different chains. The slightly larger value of $J_{\perp}$ for $\mathrm{Ca}_{2} \mathrm{CuO}_{3}$ is in line with the larger interchain distance in this compound and the somewhat higher Néel temperature.

A first remarkable conclusion from the study of the performance of the unrestricted methods is that while LDA badly fails to reproduce the magnetic coupling parameters, it does not perform bad at all for the effective hopping parameters. In comparison to DDCI3, LDA predicts $t$ 's that are about $25 \%$ smaller, whereas it results in $J$ 's that differ by an order of magnitude. In the second place, we stress that although the hybrid functionals may open a way to reach a qualitative description of all parameters at a lower cost than the DDCI3 method, the lack of a systematic, well-grounded criterion to define the optimal amount of HF exchange in the exchange functional makes this approach less interesting: all the more because this optimum amount is found to change from material to material and from property to property, e.g., the magnetic coupling parameter and the size of the band gap.

The consistency check of the parameters derived from the cluster calculations shows that the DDCI3 method performs extraordinarily well. In the first place, the DDCI3 parameters both fulfill the relation $\left(t_{1} / t_{\perp}\right)^{2}=J_{1} / J_{\perp}$ and give a reasonable value for $U$ from the relation $U=4 t_{1}^{2} / J_{1}$. In the second place, substitution of these parameters in the expressions derived by Schulz results in good approximations of the Néel temperature and the magnetic moment. None of the other methods fulfills all tests at the same time.

The periodic calculations indicate that $\mathrm{Ca}_{2} \mathrm{CuO}_{3}$ and $\mathrm{Sr}_{2} \mathrm{CuO}_{3}$ are highly ionic, although the inclusion of electron correlation introduces noticeable covalent contributions in the bonds. The analysis of the band gap shows that the top of the valence band is composed of $\mathrm{O} 2 p$ levels with important contributions from (occupied) $\mathrm{Cu} 3 d$ levels. The bottom of the conduction band, on the other hand, can be considered as an (almost) pure $\mathrm{Cu} 3 d$ band. Therefore, the nature of the gap is best described as a charge transfer gap, and our calculations do not find any evidence for the existence of an upper Hubbard band overlapping the $\mathrm{O} 2 p$ band.

\section{ACKNOWLEDGMENTS}

The authors wish to thank Ria Broer for relevant comments on the comparison with NOCI calculations and Ibério de P. R. Moreira and Carmen J. Calzado for valuable help and discussions. C. de G. acknowledges financial support through the TMR activity "Marie Curie research training grants" Grant No. FMBICT983279 established by the European Community. Financial support was provided by the "Comisión Interministerial de Ciencia y Tecnología" under CICyT Project No. PB98-1216-CO2-01 and, in part, by the "Generalitat de Catalunya" under Project No. SGR199900040. 
${ }^{1}$ H. A. Bethe, Z. Phys. 71, 205 (1931).

${ }^{2}$ J. des Cloizeaux and J. J. Pearson, Phys. Rev. 128, 2131 (1962).

${ }^{3}$ L. D. Faddeev and L. A. Takhtajan, Phys. Lett. 85A, 375 (1981).

${ }^{4}$ A. Keren, L. P. Le, G. M. Luke, B. J. Sternlieb, W. D. Wu, and Y. J. Uemura, Phys. Rev. B 48, 12926 (1993).

${ }^{5}$ T. Ami, M. K. Crawford, R. L. Harlow, Z. R. Wang, D. C. Johnston, Q. Huang, and R. W. Erwin, Phys. Rev. B 51, 5994 (1995).

${ }^{6}$ K. M. Kojima, Y. Fudamoto, M. Larkin, G. M. Luke, J. Merrin, B. Nachumi, Y. J. Uemara, N. Motoyama, H. Eisaki, S. Uchida, K. Yamada, Y. Endoh, S. Hosoya, B. J. Sternlieb, and G. Shirane, Phys. Rev. Lett. 78, 1787 (1997).

${ }^{7}$ D. A. Tennant, T. G. Perring, R. A. Cowley, and S. E. Nagler, Phys. Rev. Lett. 70, 4003 (1993).

${ }^{8}$ J. C. Bonner and M. E. Fisher, Phys. Rev. 135, A640 (1964).

${ }^{9}$ S. Eggert, I. Affleck, and M. Takahashi, Phys. Rev. Lett. 73, 332 (1994).

${ }^{10}$ O. A. Starykh, A. W. Sandvik, and R. R. P. Singh, Phys. Rev. B 55, 14953 (1997).

${ }^{11}$ M. Takigawa, O. A. Starykh, A. W. Sandvik, and R. R. P. Singh, Phys. Rev. B 56, 13681 (1997).

${ }^{12}$ J. Lorenzana and R. Eder, Phys. Rev. B 55, 3358 (1997).

${ }^{13}$ H. Rosner, H. Eschrig, R. Hayn, S.-L. Drechsler, and J. Málek, Phys. Rev. B 56, 3402 (1997).

${ }^{14}$ H. J. Schulz, Phys. Rev. Lett. 77, 2790 (1996).

${ }^{15}$ A. W. Sandvik, Phys. Rev. Lett. 83, 3069 (1999).

${ }^{16}$ V. Y. Irkhin and A. A. Katanin, Phys. Rev. B 61, 6757 (2000).

${ }^{17}$ S. G. Chung and Y. C. Chang, J. Phys. A 20, 2875 (1987).

${ }^{18}$ S. Eggert, Phys. Rev. B 53, 5116 (1996).

${ }^{19}$ N. Motoyama, H. Eisaki, and S. Uchida, Phys. Rev. Lett. 76, 3212 (1996).

${ }^{20}$ H. Fujisawa, T. Yokoya, T. Takahashi, S. Miyasaka, M. Kibune, and H. Takagi, Solid State Commun. 106, 543 (1998).

${ }^{21}$ H. Suzuura, H. Yasuhara, A. Furusaki, N. Nagaosa, and Y. Tokura, Phys. Rev. Lett. 76, 2579 (1996).

${ }^{22}$ M. Takigawa, N. Motoyama, H. Eisaki, and S. Uchida, Phys. Rev. Lett. 76, 4612 (1996).

${ }^{23}$ Z. Hiroi, M. Takano, M. Azuma, and Y. Takeda, Nature (London) 364, 315 (1993).

${ }^{24}$ Y. Tokura, S. Koshihara, T. Arima, H. Takagi, S. Ishibashi, T. Ido, and S. Uchida, Phys. Rev. B 41, 11657 (1990).

${ }^{25}$ K. Maiti, D. D. Sarma, T. Mizokawa, and A. Fujimori, Phys. Rev. B 57, 1572 (1998)

${ }^{26}$ W. E. Pickett, Rev. Mod. Phys. 61, 433 (1989).

${ }^{27}$ G. Y. Guo and W. M. Temmerman, J. Phys. C 21, L803 (1988).

${ }^{28}$ L. F. Mattheiss, Phys. Rev. B 49, 14050 (1994).

${ }^{29}$ R. L. Martin and F. Illas, Phys. Rev. Lett. 79, 1539 (1997).

${ }^{30}$ F. Illas and R. L. Martin, J. Chem. Phys. 108, 2519 (1998).

${ }^{31}$ A. Fujimori and F. Minami, Phys. Rev. B 30, 957 (1984).

${ }^{32}$ J. Zaanen, G. A. Sawatzky, and J. W. Allen, Phys. Rev. Lett. 55, 418 (1985)

${ }^{33}$ A. J. H. Wachters and W. C. Nieuwpoort, in Selected Topics in Molecular Physics, edited by E. Clementi (Chemie, Weinheim, 1972), p. 135.

${ }^{34}$ A. B. van Oosten, R. Broer, and W. C. Nieuwpoort, Chem. Phys. Lett. 257, 207 (1996).

${ }^{35}$ I. de P. R. Moreira, F. Illas, C. J. Calzado, J. F. Sanz, J.-P. Malrieu, N. Ben Amor, and D. Maynau, Phys. Rev. B 59, 6593 (1999).
${ }^{36}$ C. de Graaf, R. Broer, and W. C. Nieuwpoort, Chem. Phys. Lett. 271, 372 (1997).

${ }^{37}$ C. de Graaf, I. de P. R. Moreira, F. Illas, and R. L. Martin, Phys. Rev. B 60, 3457 (1999).

${ }^{38}$ D. Muñoz, F. Illas, and I. de P. R. Moreira, Phys. Rev. Lett. 84, 1579 (2000).

${ }^{39}$ N. Suaud and M. B. Lepetit, Phys. Rev. B 62, 402 (2000).

${ }^{40}$ A. Svane and O. Gunnarsson, Phys. Rev. Lett. 65, 1148 (1990).

${ }^{41}$ V. I. Anisimov, J. Zaanen, and O. K. Andersen, Phys. Rev. B 44, 943 (1991).

${ }^{42}$ M. T. Czyzyk and G. A. Sawatzky, Phys. Rev. B 49, 14211 (1994).

${ }^{43}$ Z. Yang, Z. Huang, L. Ye, and X. Xie, Phys. Rev. B 60, 15674 (1999).

${ }^{44}$ Y.-S. Su, T. A. Kaplan, S. D. Mahanti, and J. F. Harrison, Phys. Rev. B 59, 10521 (1999).

${ }^{45}$ Quantum-Mechanical Ab-initio Calculation of the Properties of Crystalline Materials, edited by C. Pisani (Springer, Berlin, 1996).

${ }^{46}$ R. Dovesi, R. Orlando, C. Roetti, C. Pisani, and V. R. Saunders, Phys. Status Solidi B 217, 63 (2000).

${ }^{47}$ A. D. Becke, J. Chem. Phys. 98, 5648 (1993).

${ }^{48}$ I. de P. R. Moreira and F. Illas, Phys. Rev. B 55, 4129 (1997).

${ }^{49}$ P. Reinhardt, M. P. Habas, R. Dovesi, I. de P. R. Moreira, and F. Illas, Phys. Rev. B 59, 1016 (1999).

${ }^{50}$ M. Hjorth and J. Hyldtoft, Acta Chem. Scand. 44, 516 (1990).

${ }^{51}$ R. L. Martin and P. J. Hay, J. Chem. Phys. 98, 8691 (1993).

${ }^{52}$ F. Illas, J. Casanovas, M. A. García-Bach, R. Caballol, and O. Castell, Phys. Rev. Lett. 71, 3549 (1993).

${ }^{53}$ J. Casanovas, J. Rubio, and F. Illas, Phys. Rev. B 53, 945 (1996).

${ }^{54}$ F. Illas, I. de P. R. Moreira, C. de Graaf, O. Castell, and J. Casanovas, Phys. Rev. B 56, 5069 (1997).

${ }^{55}$ I. de P. R. Moreira and F. Illas, Phys. Rev. B 60, 5179 (1999).

${ }^{56}$ P. Reinhardt, I. de P. R. Moreira, C. de Graaf, R. Dovesi, and F. Illas, Chem. Phys. Lett. 319, 625 (2000).

${ }^{57}$ N. W. Winter, R. M. Pitzer, and D. K. Temple, J. Chem. Phys. 87, 2945 (1987).

${ }^{58}$ P. W. Anderson, Phys. Rev. 115, 2 (1959).

${ }^{59}$ R. K. Nesbet, Ann. Phys. (N.Y.) 4, 87 (1958).

${ }^{60}$ R. K. Nesbet, Phys. Rev. 119, 658 (1960).

${ }^{61}$ C. de Graaf, F. Illas, R. Broer, and W. C. Nieuwpoort, J. Chem. Phys. 106, 3287 (1997).

${ }^{62}$ J. Miralles, O. Castell, R. Caballol, and J.-P. Malrieu, Chem. Phys. 172, 33 (1993).

${ }^{63}$ J.-P. Malrieu, J. Chem. Phys. 47, 4555 (1967).

${ }^{64}$ P. de Loth, P. Cassoux, J. P. Daudey, and J.-P. Malrieu, J. Am. Chem. Soc. 103, 4007 (1981).

${ }^{65}$ C. J. Calzado, J. F. Sanz, and J.-P. Malrieu, J. Chem. Phys. 112, 5158 (2000).

${ }^{66}$ V. M. García, O. Castell, R. Caballol, and J.-P. Malrieu, Chem. Phys. Lett. 238, 222 (1995).

${ }^{67}$ P.-O. Widmark, P.-A. Malmqvist, and B. O. Roos, Theor. Chim. Acta 77, 291 (1990).

${ }^{68}$ K. Pierloot, B. Dumez, P.-O. Widmark, and B. O. Roos, Theor. Chim. Acta 90, 87 (1995).

${ }^{69}$ R. Pou-Amérigo, M. Merchán, I. Nebot-Gil, P.-O. Widmark, and B. O. Roos, Theor. Chim. Acta 92, 149 (1995).

${ }^{70}$ C. J. Calzado, J. F. Sanz, J.-P. Malrieu, and F. Illas, Chem. Phys. Lett. 307, 102 (1999). 
${ }^{71}$ J. F. Sanz and J.-P. Malrieu, J. Phys. Chem. 97, 99 (1993).

${ }^{72}$ K. Andersson, M. R. A. Blomberg, M. P. Fülscher, G. Karlström, R. Lindh, P.-A. Malmqvist, P. Neogrády, J. Olsen, B. O. Roos, A. J. Sadlej, M. Schütz, L. Seijo, L. Serrano-Andrés, P. E. M. Siegbahn, and P.-O. Widmark, MOLCAS version 4, University of Lund, Sweden, 1997.

${ }^{73}$ D. Maynau and N. Ben Amor, CASDI suite of programs, Toulouse, 1997.

${ }^{74}$ L. Noodleman and E. R. Davidson, Chem. Phys. 109, 131 (1986).

${ }^{75}$ R. Caballol, O. Castell, F. Illas, I. de P. R. Moreira, and J.-P. Malrieu, J. Phys. Chem. A 101, 7860 (1997).

${ }^{76}$ K. Yosida, Theory of Magnetism, Springer Series in Solid State Sciences, Vol. 122 (Springer, Heidelberg, 1992).

${ }^{77}$ C. Adamo and V. Barone, J. Chem. Phys. 108, 664 (1998).

${ }^{78}$ C. Lee, W. Yang, and R. G. Parr, Phys. Rev. B 37, 785 (1988).

${ }^{79}$ M. J. Frisch, G. W. Trucks, H. B. Schlegel, G. E. Scuseria, M. A. Robb, J. R. Cheeseman, V. G. Zakrzewski, J. A. Montgomery Jr., R. E. Stratmann, J. C. Burant, S. Dapprich, J. M. Millam, A. D. Daniels, K. N. Kudin, M. C. Strain, O. Farkas, J. Tomasi, V. Barone, M. Cossi, R. Cammi, B. Mennucci, C. Pomelli, C. Adamo, S. Clifford, J. Ochterski, G. A. Petersson, P. Y. Ayala, Q. Cui, K. Morokuma, D. K. Malick, A. D. Rabuck, K. Raghavachari, J. B. Foresman, J. Cioslowski, J. V. Ortiz, A. G. Baboul, B. B. Stefanov, G. Liu, A. Liashenko, P. Piskorz, I. Komaromi, R. Gomperts, R. L. Martin, D. J. Fox, T. Keith, M. A. Al-Laham, C. Y. Peng, A. Nanayakkara, C. Gonzalez, M. Challacombe, P. M. W. Gill, B. Johnson, W. Chen, M. W. Wong, J. L. Andres, C. Gonzalez, M. Head-Gordon, E. S. Replogle, and J. A. Pople, Gaussian 98, Revision A.7 (Gaussian, Inc., Pittsburgh, PA, 1998).

${ }^{80}$ V. R. Saunders, R. Dovesi, C. Roetti, M. Causà, N. M. Harrison, R. Orlando, and C. M. Zicovich-Wilson, CRYSTAL98 User's Manual (University of Torino Press, Torino, 1998).
${ }^{81}$ V. R. Saunders, C. Freyria Fava, R. Dovesi, L. Salasco, and C. Roetti, Mol. Phys. 77, 629 (1992).

${ }^{82}$ Atomic basis sets are taken from the CRYSTAL program web site: http://www.ch.unito.it/ifm/teorica/Basis_Sets/mendel.html and http://www.ch.unito.it/ifm/teorica/AuxB_Sets/mendel.html

${ }^{83}$ J. M. Ricart, R. Dovesi, C. Roetti, and V. R. Saunders, Phys. Rev. B 52, 2381 (1995); 55, 15 942(E) (1997).

${ }^{84}$ Y.-S. Su, T. A. Kaplan, S. D. Mahanti, and J. F. Harrison, Phys. Rev. B 61, 1324 (2000).

${ }^{85}$ I. de P. R. Moreira, R. Dovesi, C. Roetti, V. R. Saunders, and R. Orlando, Phys. Rev. B 62, 7816 (2000).

${ }^{86}$ M. D. Towler, A. Zupan, and M. Causà, Comput. Phys. Commun. 98, 181 (1996).

${ }^{87}$ A. B. van Oosten and F. Mila, Chem. Phys. Lett. 295, 359 (1998).

${ }^{88}$ R. Broer and W. C. Nieuwpoort, Theor. Chim. Acta 73, 405 (1988).

${ }^{89}$ K. Andersson, P.-A․ Malmqvist, B. O. Roos, A. J. Sadlej, and K. Wolinski, J. Phys. Chem. 94, 5483 (1990).

${ }^{90}$ K. Andersson, P.-A. Malmqvist, and B. O. Roos, J. Chem. Phys. 96, 1218 (1992).

${ }^{91}$ F. C. Zhang and T. M. Rice, Phys. Rev. B 37, 3759 (1988); 41, 7243 (1990).

${ }^{92}$ C. J. Calzado (private communication).

${ }^{93}$ M. B. Hybertsen, E. B. Stechel, M. Schluter, and D. R. Jennison, Phys. Rev. B 41, 11068 (1990).

${ }^{94}$ R. Neudert, H. Rosner, S.-L. Drechsler, M. Kielwein, M. Sing, Z. Hu, M. Knupfer, M. S. Golden, J. Fink, N. Nücker, M. Merz, S. Schuppler, N. Motoyama, H. Eisaki, S. Uchida, M. Domke, and G. Kaindl, Phys. Rev. B 60, 13413 (1999).

${ }^{95}$ R. Weht and W. E. Pickett, Phys. Rev. Lett. 81, 2502 (1998).

${ }^{96}$ A. Shukla, M. Dolg, P. Fulde, and H. Stoll, Phys. Rev. B 60, 5211 (1999).

${ }^{97}$ R. S. Markiewicz, J. Phys. Chem. Solids 58, 1179 (1997). 